\title{
Increasing Quality of the Interframe and also extract Histogram using Super-Imposing
}

\author{
Ms K.Lavanya, Prof J.R Balakrishnan \\ ME(Computer Science and Engineering) Anand Institute Of Higher Technology \\ Professor and Director Department Of Computer Science, Anand Institute Of Higher Technology
}

\begin{abstract}
Video enhancement approach aiming to achieve high qualities in entire video. Proposes a Superimposing algorithm for improve quality of intraframe based on multiple regions-of-interest and create piecewise tone mapping curve for entire frame, interframe constraints to improve the temporal quality consistency and based on result extract histogram. Two step are used for object quality retrieval (i) ACB step improve intraframe analysis features for region-of-interest, a global tone curve is created by fusing from other region and (ii) ECB step improve interframe quality implement by histogram equalization method. Learning-based color tone mapping method to conduct global color transfer by turning the color statistic of the face region according to a per-trained set. These methods can improve the quality of some specific regions.

Keywords: Interframe, Intraframe, Super-imposing algorithm.
\end{abstract}

\section{Introduction}

Video services have become increasingly important in many areas. The quality of video service is still by several technical limitations such as poor lightening conditions, bad exposure level, and unpleasant skin color tone. This is crucial to enhance the perceptual quality of videos.

Poor lighting conditions result in low quality images. The lighting affects image quality in two aspects. The first is the brightness which is related to the signal-to-noise ratio (SNR). When there is not enough light, the captured image is dark. If one tries to brighten the image in software, it will be very noisy because of the low SNR of the captured image. Recently some video camera manufactures allow their cameras to automatically increase the camera exposure time when there is not enough light. Increasing camera exposure does improve SNR, but it degrades frame rate and causes motion blur. In addition to brightness, lighting also affects the color tone which is important for human perception. The color tone plays a critical role in the perceived look of the host and the mood of the stage.

Exposure and White Light Initialization: When the system is started, it goes to the state of "Exposure and White Light Init". The system first checks the overall intensity of the image (no face detection yet). Then the camera exposure is adjusted to ensure reasonable face brightness. Ymin as the minimal intensity value and Ymax as the maximal intensity value. If the average intensity in the face region is less than Ymin or larger than Ymax, increase or decrease the exposure level by one level at a time until the average intensity in the face region Iy falls in between Ymin and Ymax. Two issues for video enhancement.

\subsection{Multiple Region-of-interest quality Enhanced from intraframe:}

Frame may often contain multiple ROIs, it is desirable for the enhancement algorithm to achieve high intraframe quality of the entire picture where multiple ROIs can be adaptively and simultaneously enhanced.

The intraframe quality of a picture based on some pre-defined global metrics. Since these methods do not consider region differences within an image, they cannot guarantee all the important regions inside the image will be enhanced properly. Other approaches identify and improve the perceptual quality of some specific regions in an image. To perform the exposure correction based on the features of some relevant regions. A learning-based color tone mapping method to conduct global color transfer by turning the color statistic of the face region according to a pertained set. These methods can improve the quality of some specific regions, such as the face, the quality of other regions may be deteriorated.

\subsection{Interframe Quality Enhancement among Frames:}

Most of the existing enhancement state-of-the-art algorithms only focus on improving the intraframe qualities within a single frame or an image. They are not suitable for enhancing videos since the interframe quality consistencies among frames are not considered. In this super-imposing algorithms can be extended for enhancing interframe qualities under some specific applications. A learning-based method for video conferencing where frames share the same tone mapping function if their backgrounds do not change much. Although this method can achieve good interframe quality in video conferencing scenarios. 
Converging at Target and Global Illumination: Detection when there are environment illuminations changes after the system enters the state "In Target," the system needs to adjust the camera exposure and voltages accordingly. In this implemented a simple environment illumination change detector in our system. After the system enters the state "In Target," the system invokes the environment illumination change detector. At each frame, the detector computes the average intensity of the entire image including the non-face area. The detector maintains a mean value and standard deviation over time and uses the accumulated statistics to determine whether there is an environment illumination change in the new frame. If the environment illumination change is detected, the system goes back to the beginning state "Exposure and White Light Init" and starts the optimization loop.

\subsection{Video Enhancement:}

Most existing approaches have various limitations in enhancing videos. To overcome such a limitation using super-imposing algorithm, an example Figure (a) and (b) shows the enhanced results by the modified global histogram equalization algorithm and a region-based method. From Figure (c) shows that since the image is enhanced based on a global contrast metric without considering the region difference, some of the important regions such as the face are not properly enhanced. Figure (d) shows is much improved. Most existing works cannot effectively handle the interframe consistencies in a video. Although these methods may achieve proper visual qualities in each frame, the qualities among different frames.

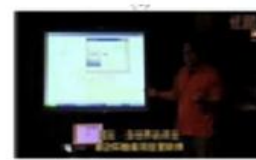

(a)

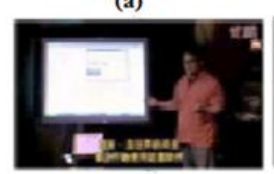

(c)

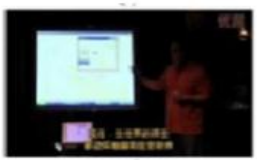

(b)

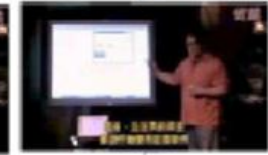

(d)

Fig 1.3.1 Enhanced Image

Fig 1.1 (a) Original Video (b) Frame Enhanced by Histogram Equalization

(c) Enhanced frame focusing on the screen region by Gaussian Mixture Model

(d) Enhanced frame by Super-imposing algorithm

\section{Related Works}

Contrast image correction method: Local contrast correction have also been proposed. Moroney uses nonlinear masking in order to perform local contrast correction. This correction can simultaneously lighten shadows and darken highlights, and it is based on a simple pixel-wise gamma correction of the input data. One of the limitations of the Moroney's algorithm common also to other local corrections is the introduction of "halo" artifacts due to the smoothing across scene boundaries and also the shrinking of the dynamic range of the scene. The adaptive histogram equalization AHE_ methods use local image information to enhance the image. In several adaptive _AHE_ techniques are reviewed and compared. The author also proposed a new AHE method based on a "modified cumulating function" that introduces two parameters.

Active lighting for video conferencing: An active lighting system which automatically adjusts the lighting so that the image looks visually appealing. The system consists of computer controllable light emitting diode light sources of different colors so that it improves not only the brightness but also the skin tone of the face. Given that there is no quantitative formula on what makes a good skin tone, they use a data driven approach to learn a good skin tone model from a collection of photographs taken by professional photographers.

Region-based color transfer from multi- reference with graph-theoretic region correspondence estimation: An automatic color transfer method based on multi-reference and graph-theoretic region correspondence estimation. When multiple high-quality reference images are available, our goal is to determine a set of best reference colors for transferring the color characteristics of the target image.

Learning-based perceptual image quality improvement for video conferencing: A learning-based technique to improve the perceptual image quality by enhancing both brightness and color tone.

The basic idea is to learn the color statistics from a training set of images which look visually appealing, and adjust the color of an input image so that its color 
statistics matches those in the training set. Conducted user study and the results show that our technique significantly improves the perceived image quality.

\section{Proposed System:}

Super-imposing algorithm will be used for the proposed system. A learning based method for video conferencing where frames share the same tone mapping function if their backgrounds do not change much. Although this method can achieve good interframe quality in video conferencing scenarios, it cannot be applied to other scenarios if the video backgrounds or contents change frequently. This method can effectively enhance both the shot change frames and the regular frames. It relies on the performance of shot detector and fails to suitably enhance the interframe quality within a shot. Better performance compare to other video. Enhance multiple ROIs within an image by segmenting or image into regions and performing color tone mapping for each region.

some specific regions, such as the face, the quality of other regions may be deteriorated.

ACB Step: object detection and saliency detection algorithms can also be adopted to obtain the ROIs. After extracting and analyzing the features from these ROIs, a global tone mapping curve is created by fusing these from different regions. if one ROI include multiple major colors, we can also view each major color region as a "sub-ROI" and pre fuse these sub-ROI features before fusing with other ROIs. Finally, the enhanced frame by this global tone mapping curve can simultaneously provide appealing qualities for different ROIs.

\section{Implementation}

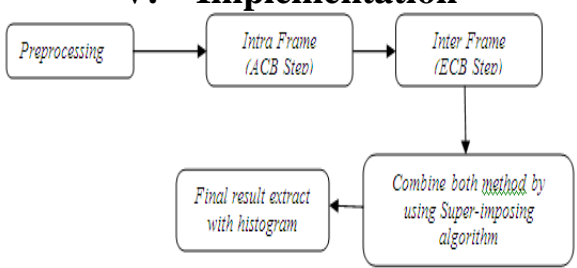

\section{Preprocessing:}

In this module input video are loaded with the help of mmreader function in matlab. And also loaded frames are converting into frames then frames are preprocessed. In preprocessing method test images. It is often desirable to be able to perform some kind of noise reduction on an image or signal. The median filter is a nonlinear digital filter technique, often used to remove noise. Such noise is reduction is a typical pre-processing step to remove the results of later pre-processing. Median filtering is very widely used in digital image processing. In the median filtering operation, the pixel value in the neighborhood window are ranked according to intensity, and the middle value(the median) becomes the output value for the pixel under evaluation.

\section{Intra frame Quality Enhancement With Multiple Regions of Interest (ROIs):}

A frame may often contain multiple ROIs, it is desirable for the enhancement algorithm to achieve high intra frame quality of the entire picture where multiple ROIs can be adaptively and simultaneously enhanced. These methods can improve the quality of

ECB step: ECB step can be implemented by the HEM-based framework.

$$
f_{g, j}^{A+E}(x)=\left(1-\lambda_{j}^{E A}\right) \cdot f_{g, j}(x)+\lambda_{j}^{E A} \cdot f_{\mathrm{prg}, j}^{A+E}(x)
$$

where $f g, j(x)$ is the intra frame global tone mapping curve from $f A+E$ preg, $j(x)$ is the tone mapping curve by the $\mathrm{A}+\mathrm{ECB}$ method in the previous frame. $\lambda \mathrm{EA}$ is the balancing parameter with the inter frame constraints embedded calculated by,

$$
\lambda_{\mathrm{EA}}=\max \left(\arg \min _{\lambda_{\mathrm{EA}}^{*}}|E(t)-E(t-1)|, \mathrm{LB}\right)
$$

where $\mathrm{E}(\mathrm{t})$ is the entropy of frame $\mathrm{t}$ and it can be calculated by

$$
E=\sum_{k}-p(k) \cdot \log p(k)
$$

here $\mathrm{p}(\mathrm{k})$ is the histogram value at bin $\mathrm{k}$. Note that a lowerbound LB is defined in to ensure that the interframe constraint can be effective in controlling the interframe consistencies. 

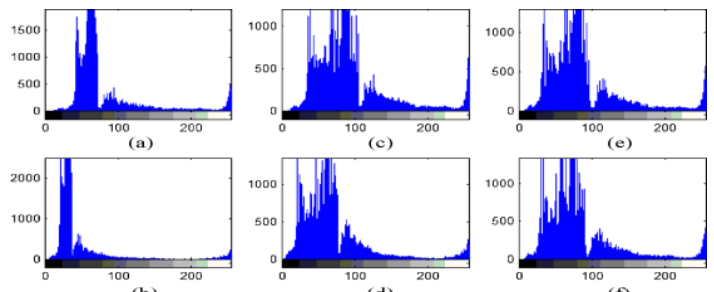

\section{Super-imposing algorithm:}

Finally combine these two method of ACB and ECB step and final result extract with histogram.

\section{Conclusion}

Proposed a new Super-imposing algorithm for video enhancement. The proposed method analyzed features from different ROIs and created a "global" tone mapping curve for the entire frame such that the quality of a frame can be properly enhanced. Furthermore, new inter frame constraints were introduced in the proposed algorithm to further improve the inter frame qualities among frames. Experimental results demonstrated the effectiveness of our algorithm.

\section{References}

[1]. T. Arici, S. Dikbas, and Y. Altunbasak, (2009) 'A histogram modification framework and its application for image contrast enhancement’, IEEE Trans. Image Process., pg no. 1921-1935.

[2]. S. Battiato and A. Bosco, (2004) 'Automatic image enhancement by content dependent exposure correction', Journal of Appl. Signal Process.,pg no. 1849-1860.

[3]. W.C. Chiou and C.-T. Hsu, (2009) 'Region-based color transfer from multi reference with graph-theoretic region correspondence estimation', IEEE Trans on Image Process., pg no. 501-542.

[4]. Z. Liu, C. Zhang, and Z. Zhang, (2007) 'Learning-based perceptual image quality improvement for video conferencing', IEEE Trans on Image Process., pg no.1035-1038.

[5]. E. Reinhard, M. Ashikhmin, B. Gooch, and P. Shirley, (2001) 'Color transfer between images', IEEE Comput. Graphics Applicat., pg no.34-41.R. Schettini and F. Gasparini, (2010) 'Contrast image correction method', Journal of Electronic Imaging, pg no.5-16.

[6]. J. A. Stark, (2000) 'Adaptive image contrast enhancement using generalizations of histogram equalization', IEEE Trans. Image Process., pg no.889-896.

[7]. M. Sun, Z. Liu, J. Qiu, Z. Zhang, and M. Sinclair, (2009) ‘Active lighting for video conferencing', IEEE Trans on Circuits Syst. Video Technol, pg no. 1819-1829.

[8]. Y. W. Tai, J. Jia, and C. K. Tang, (2005) 'Local color transfer via probabilistic segmentation by expectation-maximization', Journal in Proc. CVPR, pg no.747-754. 